by

S. Zweben, R. Maqueda, K. Hill, D. Johnson, S. Kaye, H. Kugel, F. Levinton, R. Maingi, L. Roquemore, S. Sabbagh, and G. Wurden

June 2000

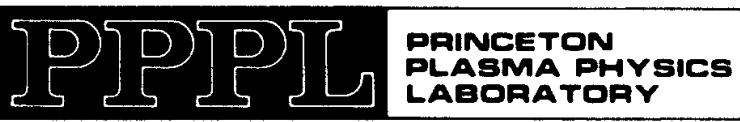

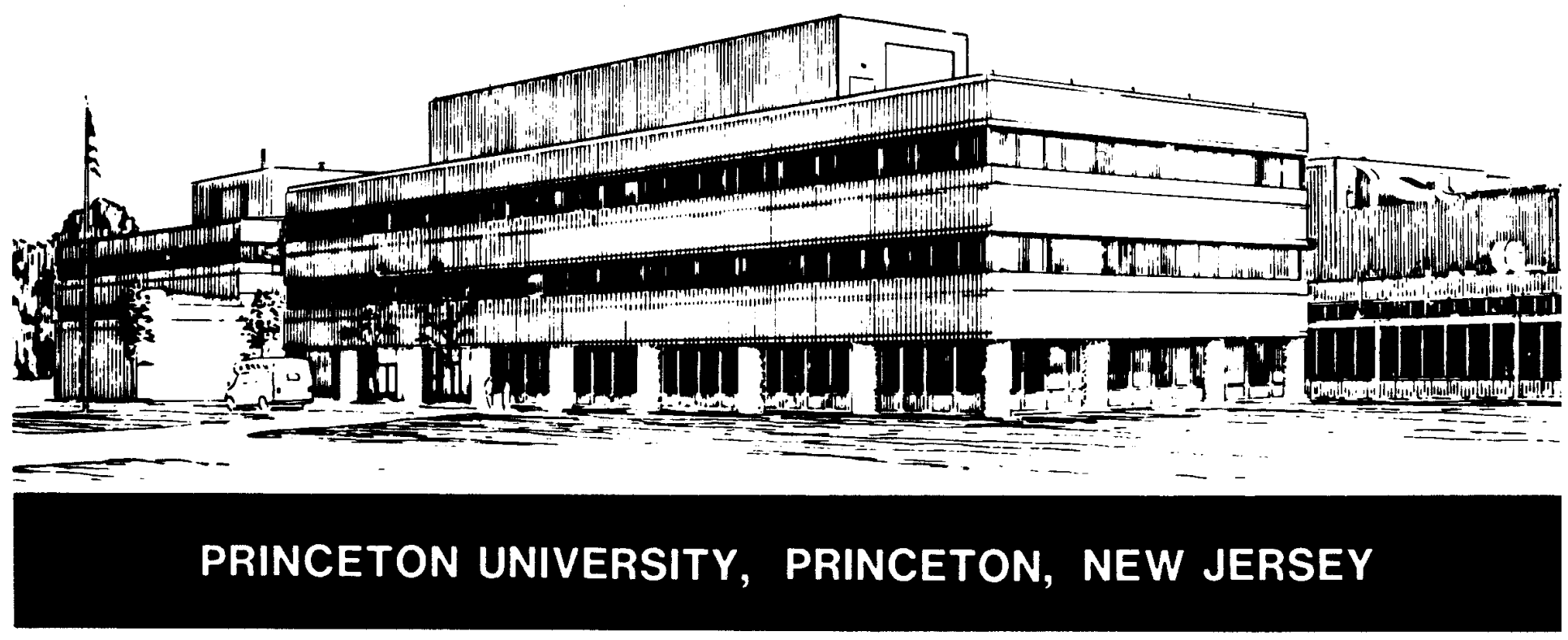




\section{PPPL Reports Disclaimer}

This report was prepared as an account of work sponsored by an agency of the United States Government. Neither the United States Government nor any agency thereof, nor any of their employees, makes any warranty, express or implied, or assumes any legal liability or responsibility for the accuracy, completeness, or usefulness of any information, apparatus, product, or process disclosed, or represents that its use would not infringe privately owned rights. Reference herein to any specific commercial product, process, or service by trade name, trademark, manufacturer, or otherwise, does not necessarily constitute or imply its endorsement, recommendation, or favoring by the United States Government or any agency thereof. The views and opinions of authors expressed herein do not necessarily state or reflect those of the United States Government or any agency thereof.

\section{Availability}

This report is posted on the U.S. Department of Energy's Princeton Plasma Physics Laboratory Publications and Reports web site in Calendar Year 2000. The home page for PPPL Reports and Publications is: http://www.pppl.gov/pub_report/

DOE and DOE Contractors can obtain copies of this report from:

U.S. Department of Energy

Office of Scientific and Technical Information

DOE Technical Information Services (DTIS)

P.O. Box 62

Oak Ridge, TN 37831

Telephone: (865) 576-8401

Fax: (865) 576-5728

Email: reports@adonis.osti.gov

This report is available to the general public from:

National Technical Information Service

U.S. Department of Commerce

5285 Port Royal Road

Springfield, VA 22161

Telephone: $1-800-553-6847$ or

(703) $605-6000$

Fax: (703) 321-8547

Internet: http://www.ntis.gov/ordering.htm 


\title{
Visible Imaging of Edge Turbulence in NSTX
}

\author{
S. Zweben, R. Maqueda ${ }^{1}$, K. Hill, D. Johnson, S. Kaye, H. Kugel, \\ F. Levinton ${ }^{2}$, R. Maingi ${ }^{3}$, L. Roquemore, S. Sabbagh ${ }^{4}$, G. Wurden ${ }^{1}$ \\ Princeton Plasma Physics Laboratory, P.O. Box 451, Princeton, NJ USA \\ ${ }^{1}$ Los Alamos National Laboratory, Los Alamos, NM USA \\ ${ }^{2}$ Fusion Physics and Technology, Torrance, CA USA \\ ${ }^{3}$ Oak Ridge National Laboratory, Oak Ridge, TN USA \\ ${ }^{4}$ Columbia University, New York, NY USA
}

\section{Introduction:}

Edge plasma turbulence in tokamaks and stellarators is believed to cause the radial heat and particle flux across the separatrix and into the scrape-off-layers of these devices. This paper describes initial measurements of 2-D space-time structure of the edge density turbulence made using a visible imaging diagnostic in the National Spherical Torus Experiment (NSTX). The structure of the edge turbulence is most clearly visible using a method of "gas puff imaging" to locally illuminate the edge density turbulence.

\section{Experimental Set-up:}

Previous measurements have shown that the visible light emission from the plasma edge as measured on a microsecond timescale has a "filamentary" structure similar to the edge density turbulence as measured with Langmuir probes [1-4]. These filaments, normally seen in $\mathrm{D}_{\alpha_{-}}$emission, are highly elongated along the magnetic field $(\lambda \gg 1 \mathrm{~m}$ long), but have a short scale length across the field in the poloidal direction $\left(\lambda_{\perp} \approx 1-10 \mathrm{~cm}\right)$. These light fluctuations are thought to be due to the rapid excitation of the neutral atoms by the electron density fluctuations, i.e., $S \propto n_{0} n_{e} f\left(T_{e}\right)$, where $S$ is the local light emission from neutrals, $n_{o}$ is the local neutral density (assumed not to fluctuate on fast timescales), $n_{e}$ is the local electron density, and $f\left(T_{e}\right)$ is normally a weak function within some range of edge temperatures. The characteristics of fluctuations in $\mathrm{S}$ and $\mathrm{n}_{\mathrm{e}}$ (as measured by a Langmuir probe) were shown to be very similar on ASDEX [2] and on the Caltech tokamak [4].

Thus we take as a working assumption that the structure of the visible light emission from the plasma edge is approximately the same as the structure of the edge density turbulence. The structure of the visible light emission in NSTX is measured using a fast framing-rate camera diagnostic, as used previously on TFTR [3]. A new feature of these measurements in NSTX is the use of "gas puff imaging" (GPI), i.e., using a local gas puff in the camera's field of view to increase the local neutral density $\mathrm{n}_{\mathrm{o}}$ and thus the brightness of the fluctuation images. Since the turbulence autocorrelation time is much faster than the framing rate of the fast camera (1 frame/msec), a supplementary measurement of the time dependence of the edge turbulence was made in NSTX using a single channel detector of light emission vs. time focused on a small area within the field of view of the camera.

\section{Initial Results:}

Several images of the visible light emission from the NSTX outer midplane edge region are shown in Fig. 1, all taken at an exposure time of $10 \mu \mathrm{sec}$ (except $20 \mu \mathrm{sec}$ for 1[b]). Fig 1(a) shows edge turbulence filaments illuminated by a deuteurium gas puff from the $60 \mathrm{~cm}$ diameter 
circular port in the middle of the image. These filaments are aligned nearly along the total magnetic field, which had $\mathrm{a} \approx 42^{\circ}$ angle to the toroidal direction at the midplane in this $\mathrm{I}=0.8$ $\mathrm{MA}, \mathrm{B}=0.3 \mathrm{~T}$ case (as evaluated from EFIT), and they have a broad range of poloidal wavelengths centered at $\lambda_{\perp} \approx 10-15 \mathrm{~cm}$. Fig. 1(b) shows a view of the same type of filaments illuminated over a wider area by the natural recycling light above the HHFW antenna $(90 \mathrm{~cm}$ high). In this case the angle of the filaments is closer to $30^{\circ}$ due to the lower plasma current $(\mathrm{I}=$ $0.5 \mathrm{MA})$. In views such as these, the filamentary structure disappears at exposure times above $\approx 100 \mu \mathrm{sec}$, and there is no repeated spatial structure from one frame to the next. Figs. 1(c,d) show the poloidal vs. radial structure of the visible light emission from a helium gas puff near the separatrix $50 \mathrm{~cm}$ above the outer midplane, as viewed along the magnetic field line using a HeI $(587.6 \mathrm{~nm})$ filter with a $10 \mu \mathrm{sec}$ exposure time. The observed spatial structure has a large variation from frame-to-frame over the $\approx 15 \mathrm{~cm} \times 15 \mathrm{~cm}$ area illuminated by the puff, such as seen in the difference between Fig.1(c) and (d). The poloidal scale of these filaments is similar to that seen in Figs. 1(a) and (b).

Fig. 2 shows an output from the single-channel light detector which viewed a $5 \mathrm{~cm}$ diameter area within the $60 \mathrm{~cm}$ diameter gas puffing port shown in Fig. 1(a). The light is measured using a PM tube with a bandwidth of $\approx 200 \mathrm{kHz}$, and sampled at $500 \mathrm{kHz}$. The raw signals, as shown in Fig. 2(a) and (b), typically have a fluctuation level of (rms/mean) $\approx 25 \%$, with a typical autocrorrelation time of $\leq 40 \mu \mathrm{sec}$, as shown in Fig. 2(d). The frequency spectrum is similar to other measurements of edge turbulence [5], as shown in Fig. 2(e). There appears to be some "bursting" in the raw signal, as shown in Fig. 2(b) and also seen in the skewed probability distribution function in Fig. 2(f). However, there are apparently no significant longtime tails of the autocorrelation function, e.g., as in Figs. 2(c) and 2(d), except during occasional low frequency coherent MHD activity.

\section{Preliminary Analysis:}

The dominant wavelength of turbulence perpendicular to the magnetic field is $\lambda_{\perp} \approx 10-15$ $\mathrm{cm}$ near the outer midplane, based on the an analysis of many poloidal vs. toroidal images such as Fig. 1(a)[7]. This is about a factor of 2-3 larger than the $\lambda_{\perp} \approx 5 \pm 2 \mathrm{~cm}$ observed for the visible light filaments on the inner limiter of TFTR at $B \approx 8 T$ [1], or the $\lambda_{\perp} \approx 6 \pm 2$ observed near the outer edge on ASDEX at $B \approx 2 \mathrm{~T}$ [2]. This suggests that the poloidal wavelength of edge turbulence is not very sensitive to the magnetic field strength, and so can not be described by a simple driftwave type expression such as $\mathrm{k}_{\perp} \rho_{\mathrm{s}} \approx 0.2$ (which is obtained for NSTX with $\lambda_{\perp} \approx 10-15$ at an assumed $\mathrm{T}_{\mathrm{e}}=25 \mathrm{eV}$ for the local $\mathrm{B}=0.15 \mathrm{~T}$ ). The signals observed in the single-channel data of Fig. 2 are similar to edge turbulence observed in other toroidal devices; for example, the frequency spectrum in Fig. 2(e) is similar to that seen with Langmuir probes in the large JET tokamak [5], and also that observed recently in Alcator C-Mod using visible light emission from a helium gas puff [6]. This reinforces the idea that the edge turbulence does not depend sensitively on the size or magnetic field of the tokamak.

The most interesting data obtained so far in NSTX are the radial vs. poloidal images such as those shown in Figs. 1(c) and (d). These images show a strong 2-D space and time modulation of the light emission with a size-scale of $\approx 10 \mathrm{~cm}$ perpendicular to the magnetic field, approximately as one would expect from viewing "end-on" the filaments seen in the poloidal vs. toroidal views of Fig. 1(a) and (b). However, the size of the light emission due to the gas puff is comparable to the scale of the turbulence, so it is difficult to characterize the size scale spectrum, except to say that there does not appear to be structure on the scale of $\approx 1 \mathrm{~cm}$ (which 
should have been resolvable in these images). In the future a poloidally extended gas puff manifold will be installed in NSTX to increase the poloidal range of this system [7].

Quantitative interpretation of these images requires a detailed model for the local relationship between the edge density fluctuations and the visible light fluctuations, which depends upon the atomic physics of the light emission process, the neutral penetration physics, and the effect of possible electron temperature and neutral density fluctuations. The turbulence wavelength sensitivity needs to be evaluated for each configuration to take into account the averaging over small wavelengths and the undersampling of large wavelengths. Finally, the possible effect of large neutral gas puffs on the edge turbulence needs to be evaluated both theoretically and experimentally. Based on this modeling, the results of visible imaging of edge turbulence can be quantitatively compared with the recent numerical models for edge turbulence [8].

[1] S.J. Zweben and S.S. Medley, Phys. Fluids B 1, 2058 (1989).

[2] M. Endler et al, Nucl. Fusion 35, 1307 (1995).

[3] R. Maqueda and G. Wurden, Nucl. Fusion 39, 629 (1999).

[4] S.J. Zweben and R. Gould, Nucl. Fusion 23, 825 (1983).

[5] M. Endler, J. Nucl. Materials 266-269 (1999) p. 84; ).

[6] J. Terry et al, 14th . Conf. on Plasma Surface Interactions (Rosenheim, 2000).

[7] R. Maqueda et al, $13^{\text {th }}$ Topical Conf. on High Temp. Plasma Diag. (Tucson, 2000).

[8] B.N. Rogers and J.F. Drake, Phys, Rev. Lett. 79, 229 (1997); F. Jenko and B.D. Scott, Phys. Plasmas 6, 2705 (1999); R.V. Shurygin, Plasma Physics Reports Vol. 25, 677 (1999); X.Q. Xu et al, Phys. Plasmas 7, 1951(2000).

This work was supported by U.S. DOE Contract DE-AC02-CHO3073. 


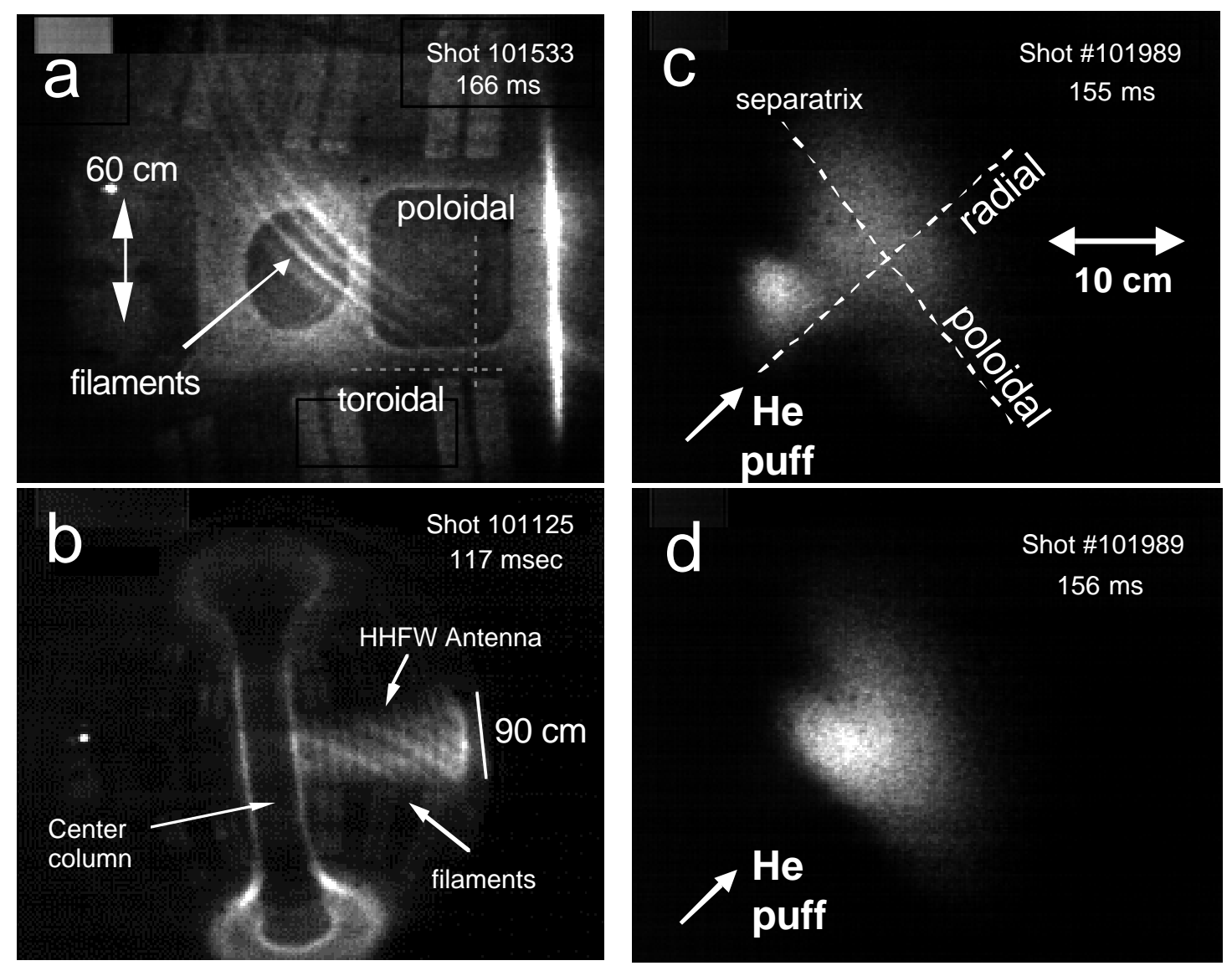

Fig. 1: Visible light images of edge plasma turbulence filaments observed in (a) a deuterium gas puff from the large circular, (b) the recycling light from the HHFW limiter, and (c),(d) two radial vs. poloidal views of a helium puff near the outer midplane, separated by $1 \mathrm{msec}$. 

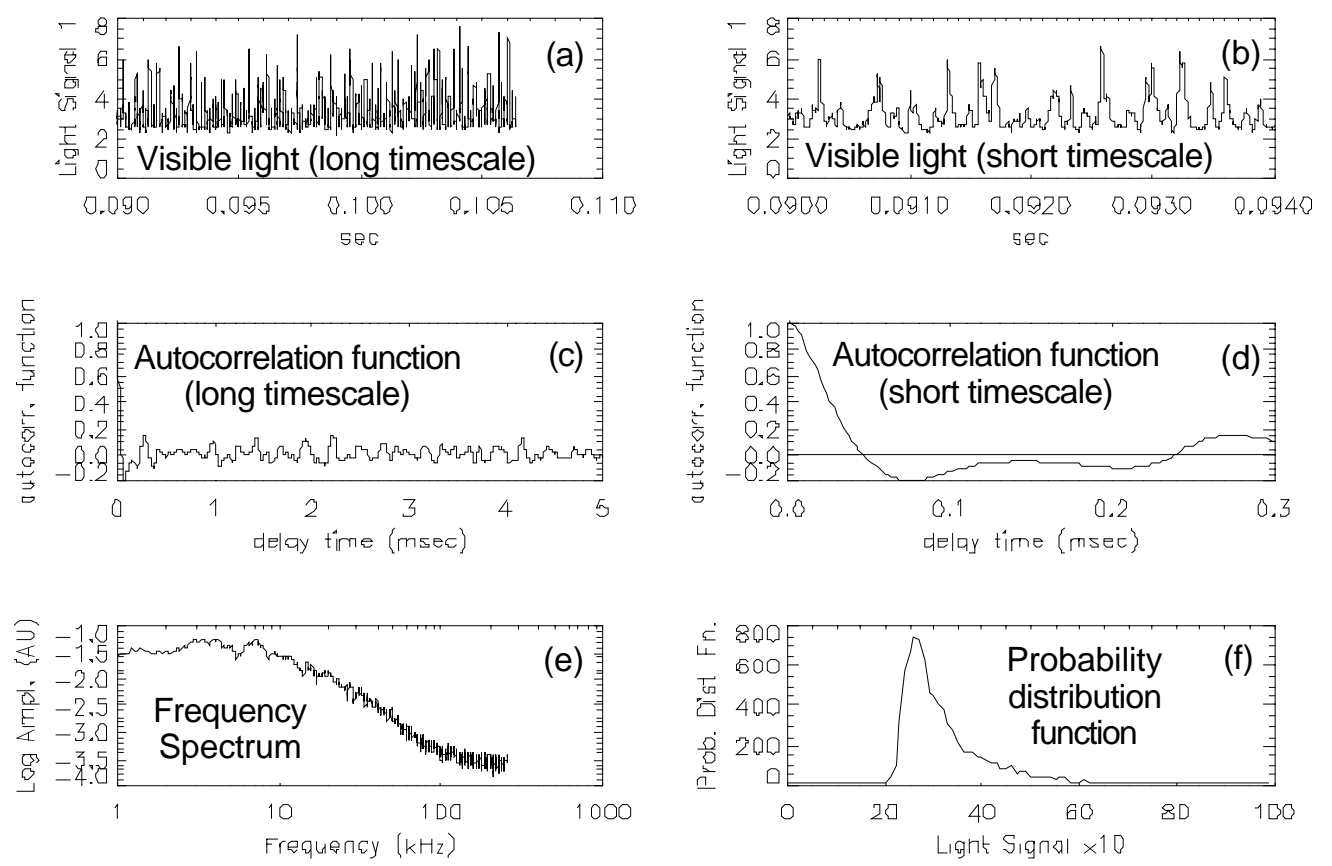

Fig. 2: Time series analysis of the visible light signal from a single-channel viewing the gas puff of Fig. 1(a). These signals are similar to edge density turbulence on other devices. 
The Princeton Plasma Physics Laboratory is operated by Princeton University under contract with the U.S. Department of Energy.

\author{
Information Services \\ Princeton Plasma Physics Laboratory \\ P.O. Box 451 \\ Princeton, NJ 08543
}

Phone: 609-243-2750

Fax: 609-243-2751

e-mail: pppl_info@pppl.gov

Internet Address: http://www.pppl.gov 